\title{
Can Fomepizole be Substituted by Abacavir in the Treatment of Methanol Poisoning?
}

\author{
Hossein Sanaei-Zadeh • Nasim Zamani • \\ Farhad Shahmohammadi
}

Published online: 12 April 2011

(C) American College of Medical Toxicology 2011

Methanol is metabolized into formaldehyde by alcohol dehydrogenase $(\mathrm{ADH})$ in the liver and then, formaldehyde is converted to formic acid by aldehyde dehydrogenase. Formic acid is responsible for the characteristic signs and symptoms of methanol toxicity such as coma, seizure, visual disturbances, and metabolic acidosis with a high plasma anion gap [1-4]. Also, due to inhibition of mitochondrial cytochrome oxidase by formate, lactic acid accumulation can occur [5-11]. Two antidotes for methanol intoxication include ethanol and fomepizole since they both are competitive inhibitors of ADH $[1,12,13]$.

Abacavir is used for treatment of human immunodeficiency virus (HIV) and acquired immune deficiency syndrome (AIDS). It is metabolized by alcohol dehydrogenase [14]. It has been shown that the metabolism of abacavir is completely inhibited by fomepizole in vitro [15]. Additionally, ethanol inhibits the metabolism of abacavir [16]. However, affinity of $\mathrm{ADH}$ for abacavir in comparison with methanol is not clear. Ghannoum and colleagues [17] presented an HIV-positive patient on the treatment with antiretrovirals, who had ingested more than 15-fold of the accepted lethal dose of pure methanol and had no signs and symptoms of methanol poisoning $11 \mathrm{~h}$ after the intake. None of the laboratory tests of the patient including arterial blood gases, measured osmolality, osmolal gap, and calculated anion gap was abnormal 5 and $11 \mathrm{~h}$ after the ingestion of methanol. Since methanol is rapidly absorbed after ingestion, its serum concentration usually reaches the peak level within 30 to $60 \mathrm{~min}$ [1], and the methanol-intoxicated patient generally

\footnotetext{
H. Sanaei-Zadeh $(\bowtie) \cdot$ N. Zamani $\cdot$ F. Shahmohammadi Department of Forensic Medicine and Toxicology,

School of Medicine (Pardis Hemmat),

Tehran University of Medical Sciences,

Tehran, Iran

e-mail: h-sanaiezadeh@tums.ac.ir
}

becomes symptomatic within 6 to $30 \mathrm{~h}$ post-ingestion $[8,9]$. With this amount of pure methanol ingested, as the authors themselves suggested [17], their patient should have become symptomatic or at least have developed metabolic acidosis within this period of time $(11 \mathrm{~h})$. They hypothesized that from the antiretroviral drugs the patient was using, only abacavir might have had a strong competitive inhibiting role of ADH. They did not administer fomepizole to their patient. We hypothesize that abacavir may be a substitute for fomepizole in the treatment of methanol toxicity. To test this hypothesis, the affinity of abacavir for ADH in comparison with methanol should firstly be experimentally determined and if it is shown that it has a greater affinity for ADH compared with methanol, a multicenter randomized controlled trial study should be designed to compare fomepizole with abacavir in the treatment of methanol-poisoned patients. Albeit, the best opportunities for conducting this study are the mass poisonings with methanol that regularly occur in developed and developing countries [9, 18-21].

Concurrent use of fomepizole and ethanol is contraindicated in the treatment of methanol poisoning because it has been shown that fomepizole prolongs the half life of ethanol. On the other hand, it is not clear if abacavir has impact on the half life of ethanol. However, it has been shown that ethanol increases the abacavir plasma concentration and not vice versa [16]. Also, fomepizole is dialyzable and the frequency of its dosing should be increased during dialysis $[12,13]$ while abacavir is poorly removed by hemodialysis [22].

As AIDS and HIV infection are worldwide problems, abacavir-used for their treatment-is also available worldwide. In contrary, methanol poisoning is a rare toxicity that is seen in the form of isolated episodes and intentional ingestion, and sometimes occurs as single epidemies [9, 18-21]. Therefore, fomepizole is not widely stocked in some hospital pharmacy stocks in comparison 
with abacavir. Additionally, its high price and lack of availability in some countries should be borne in mind.

\section{Conflict of Interest None.}

\section{References}

1. Barceloux DG, Bond GR, Krenzelok EP, Cooper H, Vale JA, American Academy of Clinical Toxicology Ad Hoc Committee on the Treatment Guidelines for Methanol Poisoning (2002) J Toxicol Clin Toxicol 40(4):415-446

2. Jacobsen D, McMartin KE (1986) Methanol and ethylene glycol poisonings. Mechanism of toxicity, clinical course, diagnosis and treatment. Med Toxicol 1(5):309-334

3. Sejersted OM, Jacobsen D, Ovrebø S, Jansen H (1983) Formate concentrations in plasma from patients poisoned with methanol. Acta Med Scand 213(2):105-110

4. Sanaei-Zadeh H, Zamani N, Shadnia S (2011) Outcomes of visual disturbances after methanol poisoning. Clin Toxicol (Phila) 49 (2):102-107

5. Nicholls P (1976) The effect of formate on cytochrome aa3 and on electron transport in the intact respiratory chain. Biochim Biophys Acta 430(1):13-29

6. Herken W, Rietbrock N, Henschler D (1969) On the mechanism of methanol poisoning. The toxic agent and influence of acid-base balance. Arch Toxikol 24(2):214-238

7. Jacobsen D, McMartin KE (1997) Antidotes for methanol and ethylene glycol poisoning. J Toxicol Clin Toxicol 35(2):127-143

8. Hovda KE, Hunderi OH, Rudberg N, Froyshov S, Jacobsen D (2004) Anion and osmolal gaps in the diagnosis of methanol poisoning: clinical study in 28 patients. Intensive Care Med 30(9):1842-1846

9. Bennet IL Jr, Cary FH, Mitchell GL Jr, Cooper MN (1953) Acute methyl alcohol poisoning: a review based on experiences in an outbreak of 323 cases. Medicine 32:431-463

10. Sefidbakht S, Rasekhi AR, Kamali K, Borhani Haghighi A, Salooti A, Meshksar A, Abbasi HR, Moghadami M, Nabavizadeh
SA (2007) Methanol poisoning: acute MR and CT findings in nine patients. Neuroradiology 49(5):427-435

11. Hantson P, Mahieu P (2000) Pancreatic injury following acute methanol poisoning. J Toxicol Clin Toxicol 38(3):297-303

12. Mégarbane B, Borron SW, Trout $\mathrm{H}$, Hantson $\mathrm{P}$, Jaeger $\mathrm{A}$, Krencker E, Bismuth C, Baud FJ (2001) Treatment of acute methanol poisoning with fomepizole. Intensive Care Med 27 (8):1370-1378

13. Brent J, McMartin K, Phillips S, Aaron C, Kulig K (2001) Fomepizole for the treatment of methanol poisoning. $\mathrm{N}$ Engl $\mathrm{J}$ Med 344(6):424-429

14. Yuen GJ, Weller S, Pakes GE (2008) A review of the pharmacokinetics of abacavir. Clin Pharmacokinet 47(6):351-371

15. Walsh JS, Reese MJ, Thurmond LM (2002) The metabolic activation of abacavir by human liver cytosol and expressed human alcohol dehydrogenase isozymes. Chem Biol Interact 142 (1-2):135-154

16. McDowell JA, Chittick GE, Stevens CP, Edwards KD, Stein DS (2000) Pharmacokinetic interaction of abacavir (1592U89) and ethanol in human immunodeficiency virus infected adults. Antimicrob Agents Chemother 44(6):1686-1690

17. Ghannoum M, Haddad HK, Lavergne V, Heinegg J, Jobin J, Halperin ML (2010) Lack of toxic effects of methanol in a patient with HIV. Am J Kidney Dis 55(5):957-961

18. Naraqi S, Dethlefs RF, Slobodniuk RA, Sairere JS (1979) An outbreak of acute methyl alcohol intoxication. Aust N Z J Med 9 (1):65-68

19. Hovda KE, Hunderi OH, Tafjord AB, Dunlop O, Rudberg N, Jacobsen D (2005) Methanol outbreak in Norway 2002-2004: epidemiology, clinical features and prognostic signs. J Intern Med 258(2):181-190

20. Paasma R, Hovda KE, Tikkerberi A, Jacobsen D (2007) Methanol mass poisoning in Estonia: outbreak in 154 patients. Clin Toxicol (Phila) 45(2):152-157

21. Paasma R, Hovda KE, Jacobsen D (2009) Methanol poisoning and long term sequelae - a six years follow-up after a large methanol outbreak. BMC Clin Pharmacol 9:5

22. Izzedine H, Launay-Vacher V, Aymard G, Legrand M, Deray G (2001) Pharmacokinetics of abacavir in HIV-1-infected patients with impaired renal function. Nephron 89(1):62-67 